\title{
Microstructural and Mechanical Profile of Carbon Fiber Epoxy Matrix Composites Containing Nanodiamonds
}

Tayyab Subhani ${ }^{1}$, Abdul Khaliq ${ }^{2}$, Umar Farooq ${ }^{3}$ and A A Khurram ${ }^{4}$

${ }^{1}$ University of Ha'il, Hail, Ha'il, Saudi Arabia, ${ }^{2}$ University of Hail, Saudi Arabia, Fawkner, Victoria, Australia, ${ }^{3}$ Institute of Space Technology, Islamabad, Islamabad, Pakistan, ${ }^{4}$ Industrial Technology Development, (NCP Complex), Islamabad, Islamabad, Pakistan

After the emergence of nanotechnology, a novel class of composites emerged as nanocomposites. Later a variant of nanocomposites appeared as multiscale composites containing at least two reinforcements at dimensionally different scales such as fibers with diameter in micrometer-range and tubes/particles/sheets with one of their dimensions at nanometer-range. Literature verifies the improvement in properties by following the strategy of incorporating multiscale reinforcements in composites [1].

In the present work, micrometer-sized carbon fibers (CFs) were incorporated along with nanometer-sized nanodiamonds (NDs) in epoxy resin to prepare a novel class of multiscale composites. The composites with three different loadings of NDs, i.e. $0.2 \mathrm{wt} \%, 0.4 \mathrm{wt} \%$ and $0.8 \mathrm{wt} \%$ were prepared by vacuum assisted resin transfer molding along with a reference composite without NDs. Carbon fabric had high strength polyacrylonitrile based 2D-woven carbon fibers in 3K-plain weave. NDs were $\mathrm{UV} / \mathrm{O}_{3}$ treated to improve their dispersion in epoxy resin. Detailed experimental procedure is available elsewhere [1].

Figure 1(a) shows carbon fiber epoxy matrix composite containing carbon fibers in the form of 2D-woven fabric embedded in epoxy resin while Figure 1(b) reveals the surface morphology of an individual carbon fiber before impregnating with epoxy resin. Figure 1(c) is the micrograph of NDs in as-received condition, i.e. before de-agglomerating, $\mathrm{UV} / \mathrm{O}_{3}$ treating and dispersing in epoxy resin. Figure 1(d) shows individual carbon fiber in epoxy matrix composite without NDs; the fragments of cured epoxy are visible. Figure $1(\mathrm{e})$ and (f) are the images of individual carbon fibers taken from the composites containing $0.4 \mathrm{wt} \%$ and $0.8 w t \%$ NDs. A good dispersion of NDs can be seen in composites containing 0.4wt\% NDs while NDagglomerates are evident after increasing the contents of NDs to $0.8 \mathrm{wt} \%$.

The incorporation of NDs in CF-epoxy composites resulted in an increase in tensile and flexural properties. The addition of $0.2 \mathrm{wt} \%$ NDs enhanced tensile strength and modulus values to $964 \pm 40 \mathrm{MPa}$ and $85 \pm 3 \mathrm{GPa}$ from $895 \pm 35 \mathrm{MPa}, 83 \pm 5 \mathrm{GPa}$ in composites without NDs, respectively (Figure 2(a and b)). Further rise in tensile properties $(1030 \pm 42 \mathrm{MPa}$ and $98 \pm 5 \mathrm{GPa})$ was observed by increasing NDs content $(0.4 \mathrm{wt} \%)$ and also because of their uniform distribution in epoxy matrix (Figure 1(e)). However, still further rise in the content of NDs resulted in a decrease in tensile properties, i.e. $980 \pm 78 \mathrm{MPa}, 92 \pm 7 \mathrm{GPa}$, respectively. This may be attributed to the presence of ND-agglomerates (Figure 1(f)) acting as defects, as also observed in other studies [2]. Nevertheless, tensile properties obtained in composites containing $0.8 \mathrm{wt} \%$ NDs are still higher than the composites without NDs. A similar trend in flexural strength of composites containing NDs was observed as found in tensile properties (Figure 2(c) and (d)), i.e. composites containing $0 \mathrm{wt} \%, 0.2 \mathrm{wt} \%, 0.4 \mathrm{wt} \%$ and $0.8 \mathrm{wt} \%$ NDs had values of $450 \pm 6 \mathrm{MPa}, 489 \pm 8 \mathrm{MPa}$, $513 \pm 10 \mathrm{MPa}$ and $497 \pm 14 \mathrm{MPa}$, respectively. However, an insignificant effect on flexural modulus values was observed in composites with three loadings of NDs and the one without the addition of NDs, i.e. $50 \pm 5 \mathrm{GPa}, 51 \pm 6 \mathrm{GPa}, 52 \pm 7 \mathrm{GPa}$ and $50 \pm 8 \mathrm{GPa}$.

Generally, the trend observed in composites containing NDs is similar to that reported in literature [3]: an increase in properties up to a certain quantity of NDs, followed by a decrease after further addition. The 
nanoreinforcements with different physical and chemical morphologies such as carbon nanotubes have shown a different set of properties in comparison to composites containing NDs with same loading fractions [4]. MWCNTs act as nanofibers and have the potential to increase tensile and flexural properties more than NDs, which simply act as nanoparticles in composites. Crack deflection, bridging, pinning and pull-out phenomena may be more effective in the presence of MWCNTs in composites than NDs. In contrast, NDs have the potential to increase the hardness of composites, which increases the strength but pronounced effect of the increased hardness of composites containing NDs appear in better tribological properties such as wear resistance and friction coefficient [2].
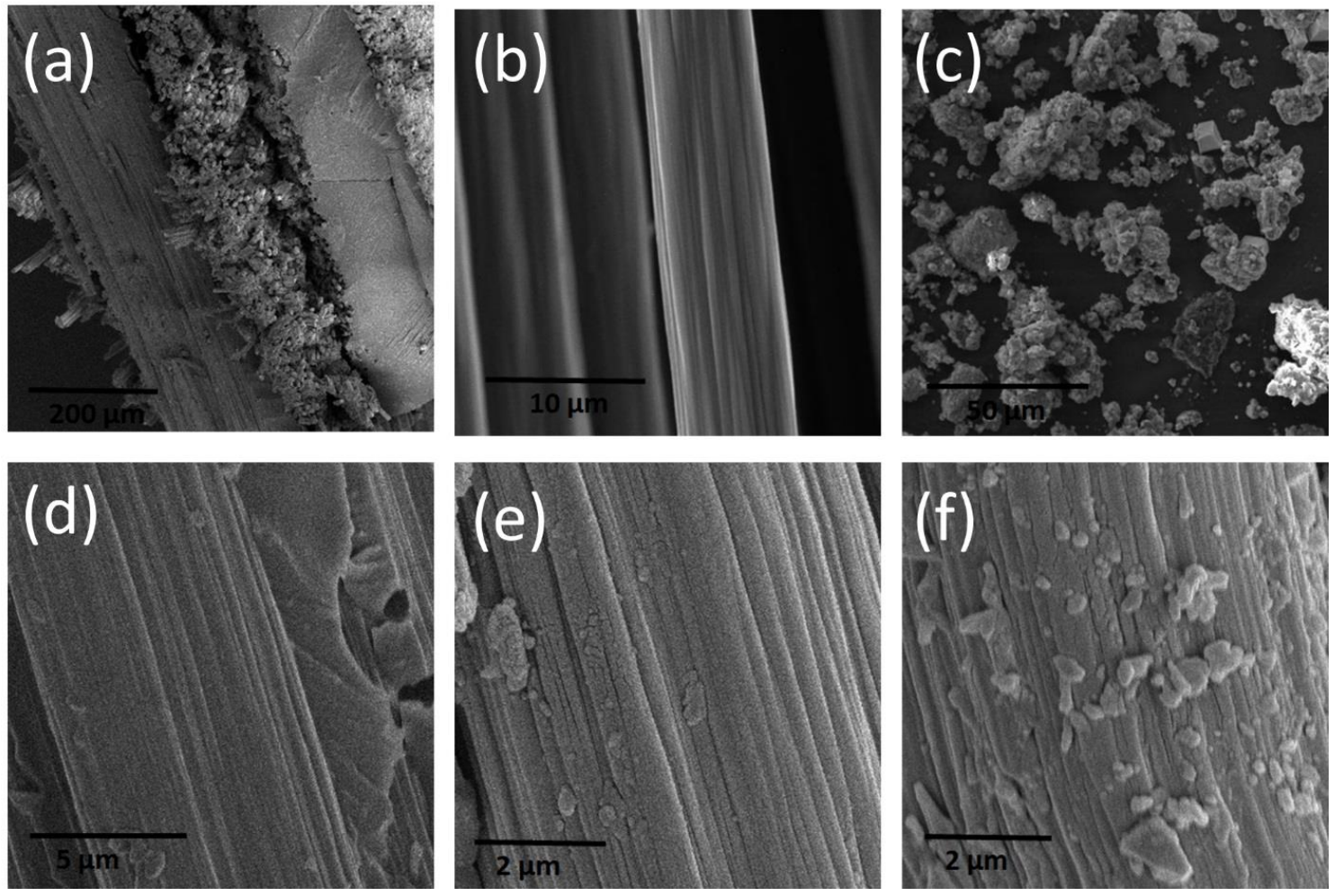

Figure 1. (a)Two-dimensionally woven structure of carbon fiber epoxy matrix composite containing nanodiamonds. (b) Individual carbon fiber before impregnating with epoxy resin. (c) Nanodiamonds in as-received condition. (d) Individual carbon fiber embedded in epoxy resin. (e) Individual carbon fiber in composite containing $0.4 \mathrm{wt} \%$ nanodiamonds. (f) Individual carbon fiber in composite containing $0.8 \mathrm{wt} \%$ nanodiamonds. 

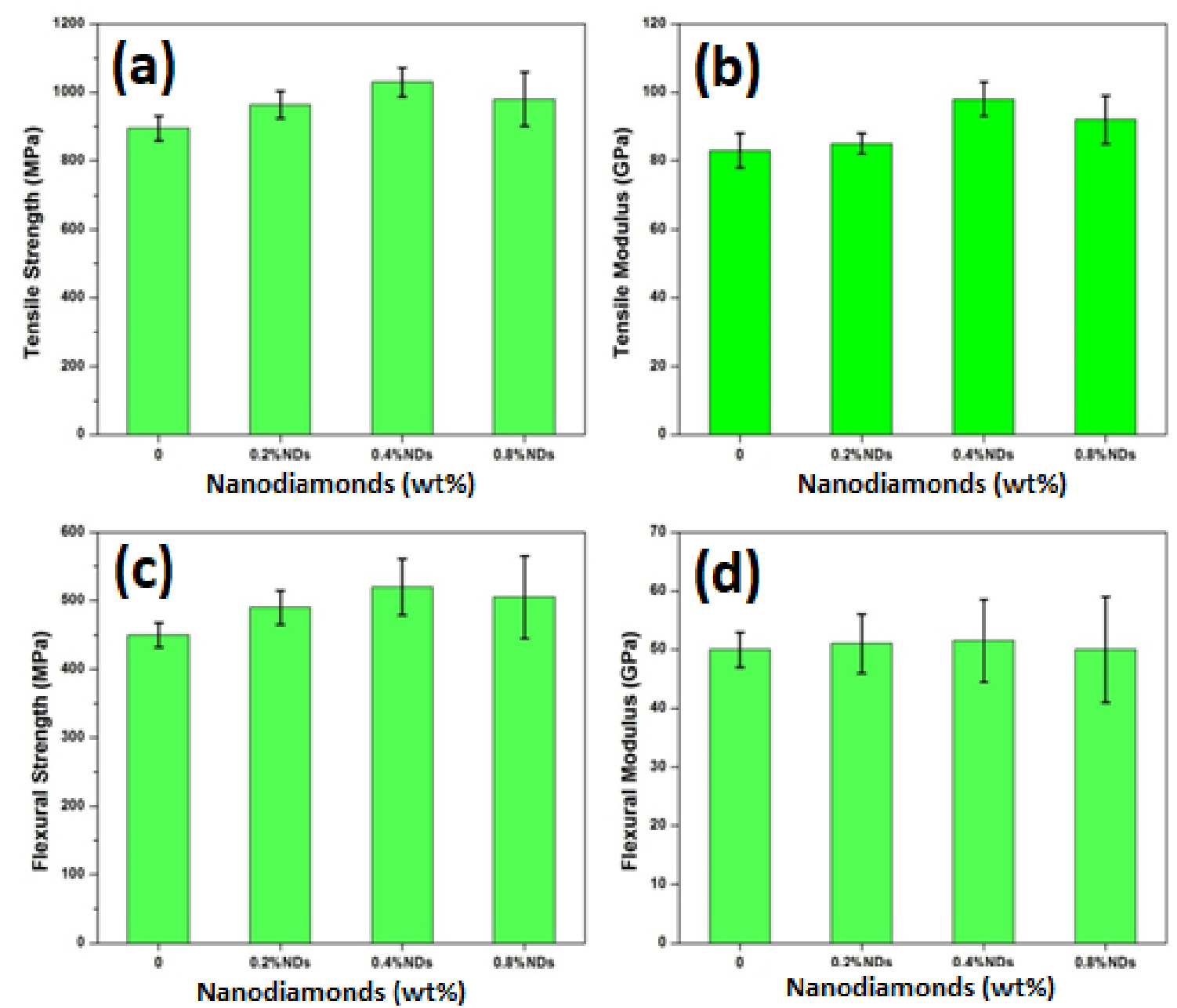

Figure 2. Tensile and flexural properties of carbon fiber epoxy matrix composites without and with $0.2 \mathrm{wt} \%, 0.4 \mathrm{wt} \%$ and $0.8 \mathrm{wt} \%$ nanodiamonds: (a) tensile strength, (b) tensile modulus, (c) flexural strength and (d) flexural modulus.

\section{References}

[1] Zaheer U et al, Composite Interfaces, 25 (2018), p. 1005-1018.

[2] Subhani T et al, Materials \& Design, 87 (2015), p. 436-444.

[3] Zaheer U, Khurram A A, Subhani T, Advanced Composites and Hybrid Materials, 1 (2018), p. 705721.

[4] Subhani T et al, Polymer Composites, 38 (2017), p. 1519-1528. 